\title{
Research on Wavefront Curvature and Motion Compensation by means of the Spotlight SAR
}

\author{
Su Fu-lin, Li Jun*, Gao Jian-jun and Sun Jing
}

Harbin Institute of Technology, Department of Information Engineering, Harbin Institute of Technology, No. 92, West DaZhi Street Harbin 150001, China

\begin{abstract}
Spotlight SAR mode can achieve high resolution radar imaging, but the measurement error, radar platform motion and the other reasons, will make the image suffer the severe defocus and even distortion. This paper introduces the range alignment technology of ISAR imaging to Spotlight SAR imaging,and improved the MD-PGA algorithm, so as to make motion compensation for the error.Afterwards, with the improved Space-variant post filtering method, compensate the wavefront curvature error to improve the image quality. Finally the processing results of measured data showed the effectiveness of this method.
\end{abstract}

Keywords: Spotlight SAR, polar format algorithm, motion compensation, wavefront curvature correction.

\section{INTRODUCTION}

Spotlight synthetic aperture radar is a kind of important SAR working mode, in the target data acquisition, the radar beam illuminates the target area all the time, getting longer synthetic aperture time to improve azimuth resolution [1]. In order to obtain a high resolution SAR image efficiently, the researches about motion compensation to improve the image quality are paid more manpower and given more attention both at home and abroad something is missing. The main methods of motion compensation are as follows: phase gradient autofocus algorithm (PGA) [2-7] and the motion field algorithm based on Doppler rate estimation, such as MapDrift (MD) [8-12], the algorithms based on image standards, and all kinds of improved algorithms about motion compensation [13] and so on.

This paper first introduces the PFA algorithm [14-18] of high resolution imaging, and then introduces the range alignment technology of ISAR for motion compensation, furthermore, improves the MD - PGA algorithm to have better results in motion compensation. After the application in processing and analyzing the measured data, the theory of algorithm is verified feasible and helpful.

\section{IMAGING PRINCIPLE OF PFA ALGORITHM}

Based on the geometry model of spotlight SAR data acquisition shown in Fig. (1), suppose that $p\left(x_{t}, y_{t}, 0\right)$ is a target point in the scene area, the synthetic aperture time is $T_{a}$, the

*Address correspondence to this author at the Harbin Institute of Technology, Department of Information Engineering, Harbin Institute of Technology, No. 92, West DaZhi Street Harbin 150001, China; Tel: 15845007221;

E-mail: lijun_hit@163.com carrier frequency is $f_{c}$, the pulse width is $T_{p}$, the chirp rate of wideband FM signal is $k$, while $\mathrm{t}$ and $\tau$ are the slow and fast time respectively. So then we can get the echo signal of the point $p$ as below:

$$
\begin{aligned}
s_{r}(t, \tau)= & \operatorname{rect}\left(\frac{t}{T_{a}}\right) \operatorname{rect}\left(\frac{\tau-2 R_{t} / c}{T_{p}}\right) \\
& \cdot \exp \left\{j 2 \pi\left[f_{c}\left(t-\frac{2 R_{t}}{c}\right)+\frac{1}{2} k\left(\tau-\frac{2 R_{t}}{c}\right)^{2}\right]\right\}
\end{aligned}
$$

Where $R_{t}$ and $R_{a}$ are the distances, from antenna phase center to the point $p$, and the center of the scene respectively. Make demodulation processing for echo signal and convert it to the frequency domain in range direction, and then pulse compression processing can be done to get the result as follows:

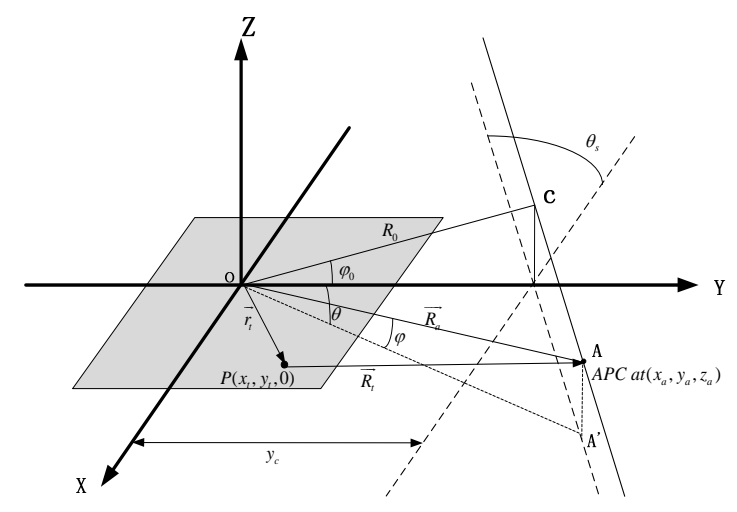

Fig. (1). Geometry model of spotlight SAR data acquisition. 


$$
S_{r M}\left(t, f_{\tau}\right)=\operatorname{rect}\left(\frac{t}{T_{a}}\right) \operatorname{rect}\left(\frac{f_{\tau}}{k T_{p}}\right) \exp \left[j\left(K_{r}\left(R_{a}-R_{t}\right)\right)\right]
$$

Where $K_{r}=4 \pi\left(f_{c}+f_{\tau}\right) / c$ is the size of the radial wavenumber. For further analysis, make plane wavefront approximation, ignoring the effect of amplitude information. The projections of the wavenumber vector, in azimuth and range directions are represented by $K_{x}=K_{r} \cos \varphi \sin \theta$, $K_{y}=K_{r} \cos \varphi \cos \theta$ respectively. In the wavenumber domain, formula (2) can be indicated as below:

$S_{r M}\left(K_{x}, K_{y}\right)=\exp \left[j\left(x_{t} K_{x}+y_{t} K_{y}\right)\right]$

It can be seen from the above equation that, there is a direct relationship between the echo signal representation and the target position's information, which can obtain target position's information by two-dimensional Fourier transform. The improvement of the computational efficiency is generally implemented through two times of FFT transform, which requires that the processed signal must satisfy the characteristic of uniform sampling in wavenumber domain. However, the real echo signal is arranged with polar format, in the wavenumber's frequency domain. While through twodimensional interpolation, it can be converted into the rectangular format arrangement.

\section{MOTION COMPENSATION ALGORITHM}

Due to the interference of external environment,and the imprecision of its control system when capture data, the radar platform cannot maintain the ideal state of motion, leading to echo signal's deviation of phase in azimuth, which needs for motion compensation processing, in the imaging procedure to correct the phenomenons of defocus and distortion caused by it, just in order to improve the final image quality.

The platform's unideal motion mainly consists of two aspects [19-21]: Deviations from the ideal track translational motion and the posture rotation of the platform's own. The latter influence can be ignored after the inertial navigation system's (INS) control, while the former effect can not be removed completely, which needs compensation for imaging errors that its causes. Here will mainly study the motion compensation based on echo data.

\section{RANGE ALIGNMENT ALGORITHM}

Range alignment is also called the envelope alignment $[22,23]$ - a key technology of ISAR imaging. By eliminating the adjacent echo's dislocation in the range direction, realize the envelope alignment of the one dimensional range profile, to eliminate the phase error caused by the range translation. The premise of its use is that the envelopes of adjacent echo signals, received in data acquisition have strong similarities. Spotlight SAR is generally for small scene and high resolution imaging, moreover, the rotational angle of carrier aircraft, corresponding to adjacent echoes, is generally less than $0.01^{\circ}$, so the scattering point's walk is very small, making the real envelopes of adjacent echo very similar, which is the key factor to introduce range alignment technique to Spotlight SAR imaging.

This paper mainly uses the envelope correlation method and the accumulated envelope correlation method, to implement them and contrast the imaging results. Correlation method is widely used as a range alignment algorithm, mainly based on the similarities between adjacent echoes in the domain of one-dimensional range, using the crosscorrelation method that compensates translational motion based on the delay corresponding to adjacent echoes' peaks, to obtain better alignment for adjacent envelope. A certain fixed one of the echo signal is set as the reference signal, and generally we choose the first echo signal or the one from Synthetic aperture Center. This algorithm has high alignment accuracy, but with accumulation of phase errors inevitably, leading to the phenomena of envelope's drift and envelope's jumpy-changing. While the accumulated envelope correlation method sets the weighted sum of all the previous aligned echoes' one-dimensional range profiles. The single echo's error has little effect on the processing, so the phenomena of sudden jumpy-changing caused by the error accumulation is greatly reduced.

In reference [23], one global optimal method is proposed that based on amplitude correlation of all the echoes, and it is possible to formulate the process as follows:

$$
\begin{aligned}
\left(\tau_{2}, \tau_{3}, \ldots, \tau_{M}\right)= & \arg \max _{\tau_{2}, \tau_{3}, \ldots, \tau_{M}} \sum_{i=1}^{M} \sum_{j=i+1}^{M}\left|S_{i}\left(n-\tau_{i}\right)\right| \\
& \cdot\left|a(i) S_{j}\left(n-\tau_{j}\right)\right|
\end{aligned}
$$

Where $M$ represents the times of echo, and $a(j)$ represents the weighted coefficient.

When determining the weighted coefficient, consider that the closer echo with the fixed echo, has stronger correlation, so we often set bigger weights for the closer adjacent echoes. However, if we want to eliminate the jumpychanging errors' distance image, we need to set the weights corresponding to the closer adjacent echo to be smaller. Combined, the weighted coefficients are generally set to be 1 uniformly.

\section{MD-PGA AUTOFOCUS ALGORITHM}

The MD-PGA algorithm [24] is just an autofocus algorithm combining PGA and MD algorithm. Consider dividing the whole aperture into multiple sub apertures, and then image the sub aperture, and estimate the phase error of each sub aperture with PGA algorithm. While the linear phase error between the sub aperture images, focused with PGA is estimated using MD algorithm. After that, piece together the phase error of each sub aperture and the linear phase error between the sub apertures, making the phase error of the 


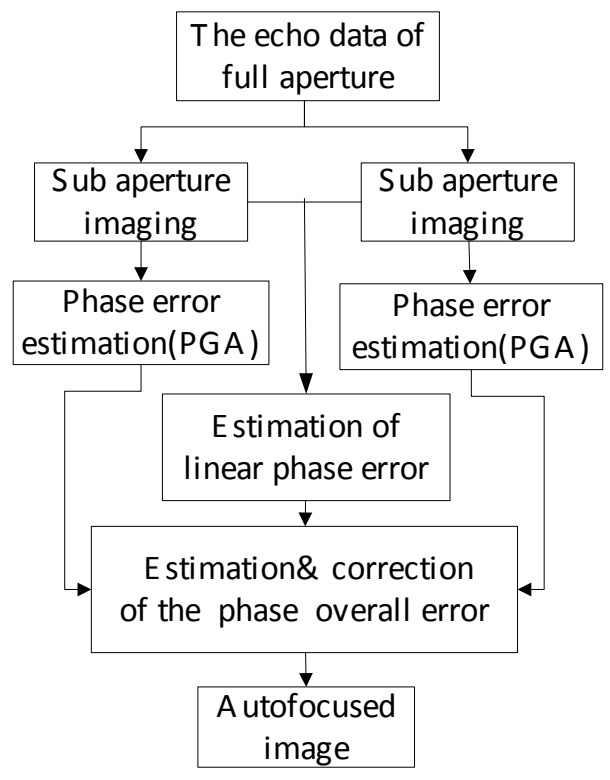

Fig. (2). Original MD-PGA process.

whole aperture is continuous. The MD-PGA process when divided into two sub apertures is showed as Fig. (2), and the process of multiple sub apertures is similar.

According to the analysis of the original MD-PGA process, it can be known that the influence of the linear phase error is reduced, but there are still some defects without estimation and compensation on high-order phase error of the adjacent sub apertures. This paper improved the original MD-PGA algorithm, and the improved flow diagram is shown in Fig. (3). Process the imaging results of the full aperture echo data with PGA algorithm, followed with phase error estimation and phase correction of echo data, and the full aperture data after phase correction is divided into several sub apertures to image. Then estimate the linear phase error between the sub aperture imaging results using MD algorithm, after that, combine the linear phase error estimated by MD algorithm and the full aperture imaging phase error estimated with PGA to construct a new correction factor of the phase error. Finally correct the phase error of the full aperture echo data with the new correction factor, and the focused image is obtained. The improved MD-PGA algorithm can not only correct the linear phase error of the original PGA process, but also compensate the high-order phase error between the sub apertures.

\section{CORRECTION OF WAVEFRONT CURVATURE ER- ROR}

In section2, the derivation of PFA imaging principle in the process, takes the planar wavefront assumption. But when the imaging area is too broad, the error brought by planar wavefront assumption can't be ignored, cause the reduced imaging distance and the need of high resolution,

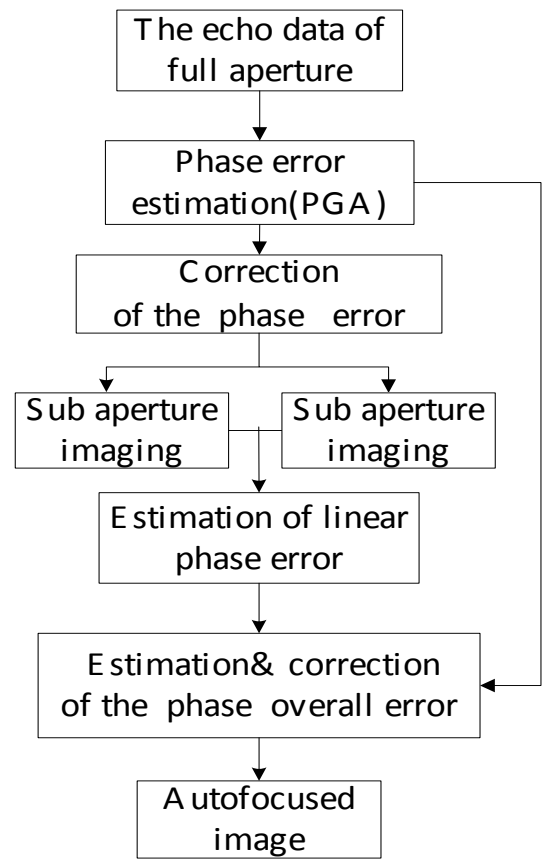

Fig. (3). Improved MD-PGA process.

require correction. Considering the compensation of the wavefront curvature error, this paper makes some improvements on the method of Space-variant post processing, and the algorithm deals with the PFA imaging result directly, with the correction of wavefront curvature in the complex image domain.

Makes two-dimensional Taylor series expansion for the echo data's expression (3) directly in wavenumber domain, and the formula below can be obtained.

$S_{r M}\left(K_{x}, K_{y}\right)=\exp \left[j k_{r}\left(R_{a}-R_{t}\right)\right]=\exp \left[j \frac{\sqrt{K_{x}^{2}+K_{y}^{2}}}{\cos \varphi}\left(R_{a}-R_{t}\right)\right]_{(5}$
$=\exp \left\{j\left[\begin{array}{l}c_{01} K_{x}+c_{10}\left(K_{y}-K_{c}\right)+c_{11} K_{x}\left(K_{y}-K_{c}\right)+c_{20} K_{x}^{2} \\ +c_{02}\left(K_{y}-K_{c}\right)^{2}+\cdots\end{array}\right]\right\}$

Where $K_{c}=\frac{4 \pi}{c} f_{c} \cos \varphi_{0}$ is the bias of wavenumber $K_{y}$ in

$\mathrm{Y}$ direction. The specific derivation process is described in [25], and the coefficient values can be calculated with Taylor expansion formula. From the formula (5) can know that the influence of wavefront curvature mainly are: a). defocus and blur in the azimuth caused by quadratic phase error, which can be corrected by two-dimensional spatial filter. Consider that the defocusing phenomena mainly occur in azimuth direction, the spatial filtering is only designed for onedimensional azimuth filtering. b). geometric distortion of the target's position caused by first-order linear phase error, which can be corrected with resampling in the spatial domain. The process is illustrated in Fig. (4). 


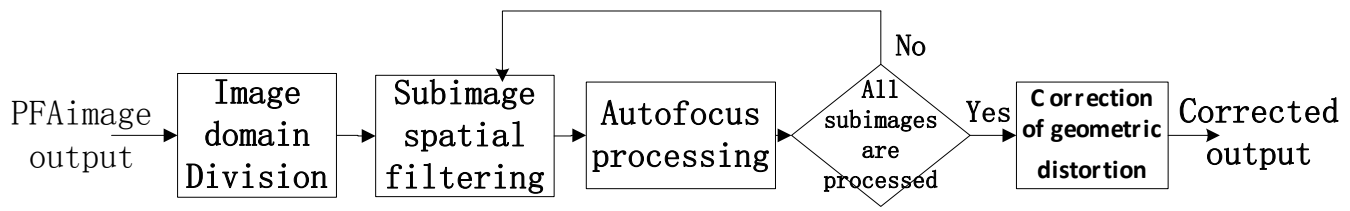

Fig. (4). Space-variant post processing.

Table 1. Parameters of real data.

\begin{tabular}{|c|c|c|c|c|c|c|}
\hline $\mathbf{T}_{\mathbf{P}}$ & $B_{r}$ & $f_{s}$ & $f_{c}$ & $f_{P R F}$ & $T_{s}$ \\
\hline \hline $5 \mu s$ & $200 \mathrm{MHZ}$ & $480 \mathrm{MHZ}$ & $17.15 \mathrm{GHZ}$ & $1000 \mathrm{HZ}$ & $56 \mathrm{~s}$ & $151 \mathrm{HZ}$ \\
\hline
\end{tabular}

The high resolution imaging general flowchart of spotlight SAR is shown in Fig. (5).

\section{EXPERIMENTAL RESULTS}

In this paper, the measured data processed are the echo data from airborne spotlight SAR acquisition, and the specific parameters are shown in Table 1. We chose 2048 pulses'echo from the full aperture to process. The corresponding squint angle is $0.3348 \mathrm{rad}$, while the range resolution is $0.75 \mathrm{~m}$ and the azimuth resolution is about $0.4 \mathrm{~m}$.

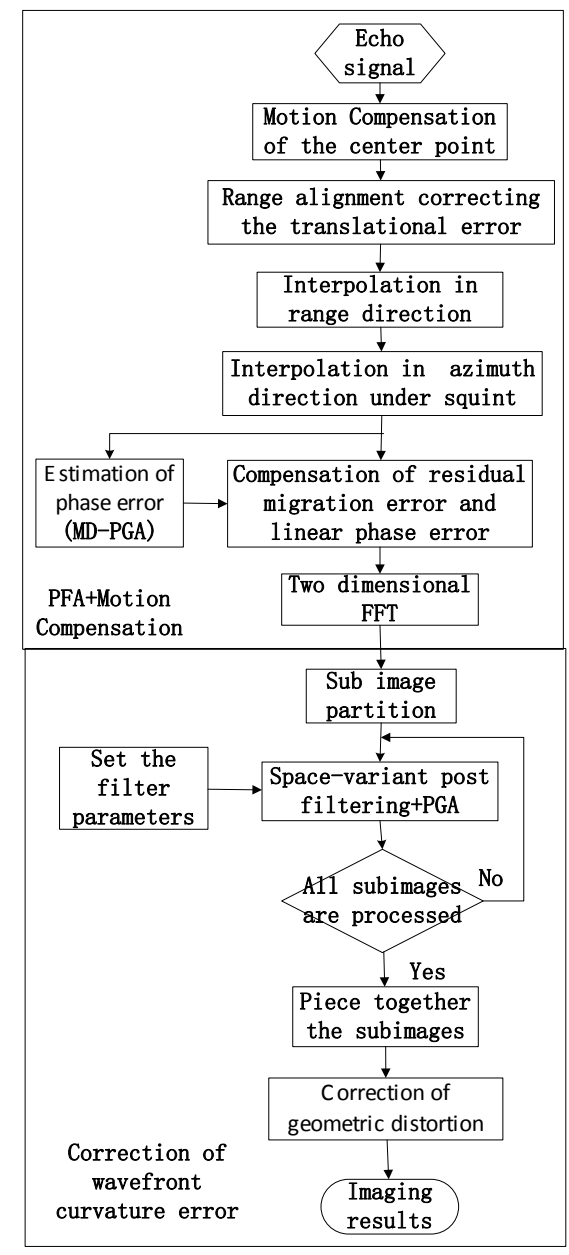

Fig. (5). Spotlight SAR imaging processing flow chart.

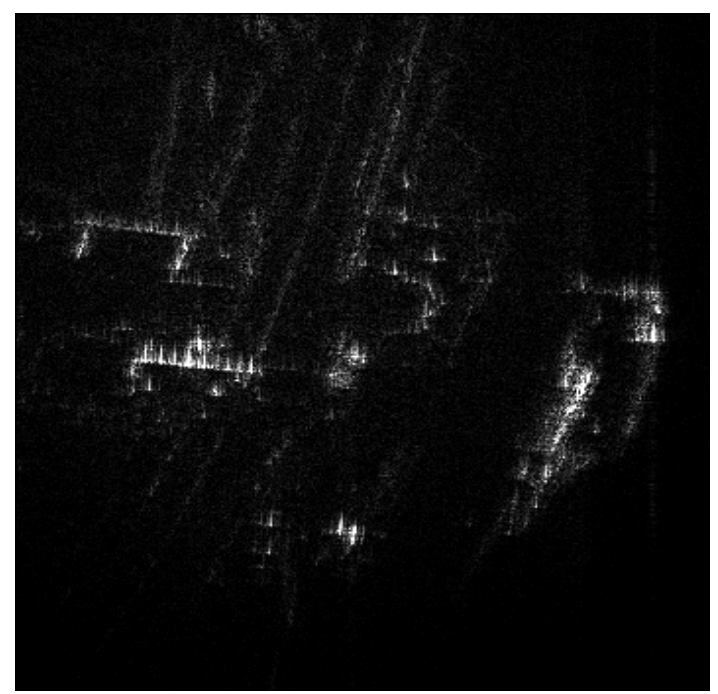

Fig. (6). Directly imaging.

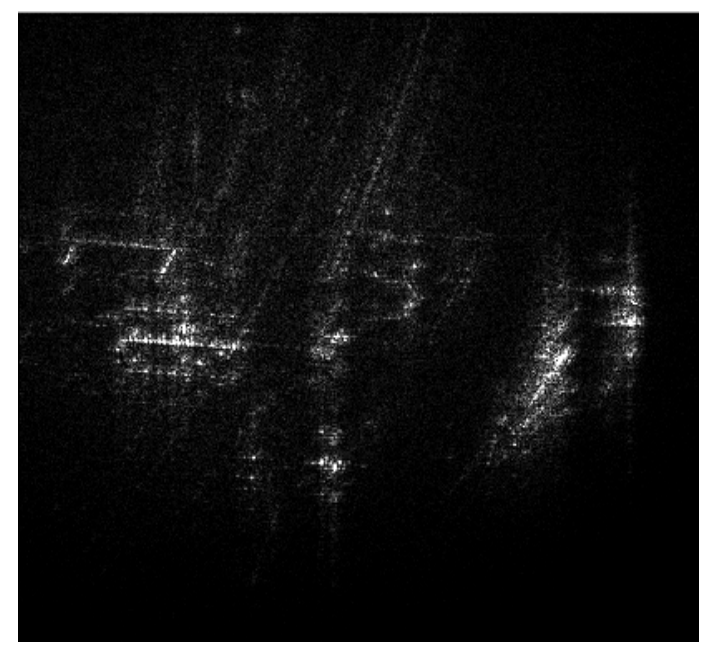

Fig. (7). Envelope correlation alignment result.

Fig. (6) is the directly imaging result without motion compensation, looking fuzzy. After using the envelope correlation method and the global accumulated envelope correlation method to achieve the range alignments, the imaging results are shown in Fig. (7) and Fig. (8) respectively. From the imaging results, it can be obviously seen that the imaging 


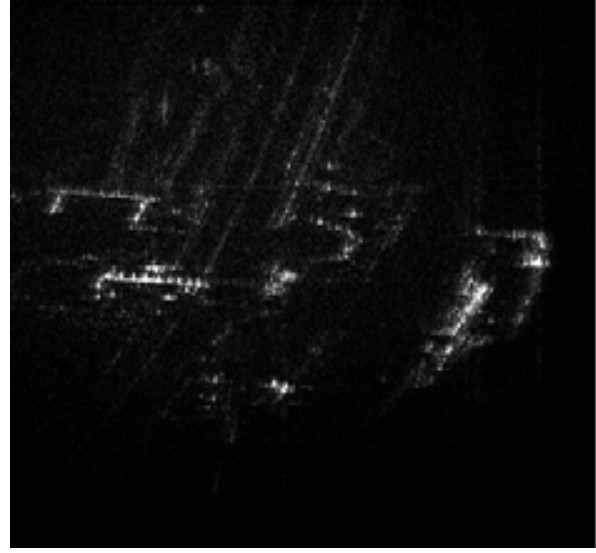

Fig. (8). Accumulated envelope correlation alignment result.

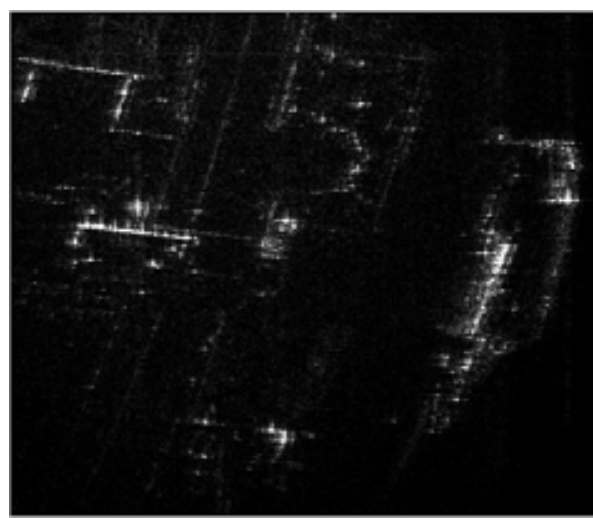

(a) MD-PGA

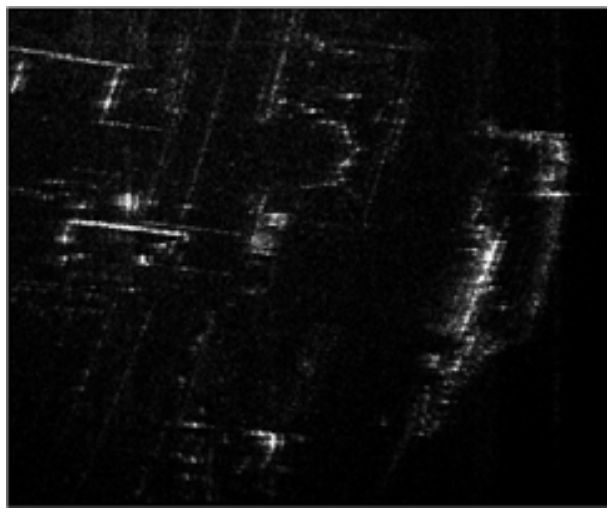

(b) Improved MD-PGA

Fig. (9). Autofocus imaging results.

result in Fig. (8) focuses better than in Fig. (6), and the result has been significantly improved; While there are serious false contours and defocused phenomenon in Fig. (7). Then we evaluate the images objectively from the aspects of image entropy and contrast ratio. Fig. (6) - entropy: 11.3927, contrast ratio: 26.4728; Fig. (7) - entropy: 11.607, contrast ratio : 23.4534; Fig. (8) - entropy: 11.0467, contrast ratio: 33.8693. It can be known that compared to the formers,

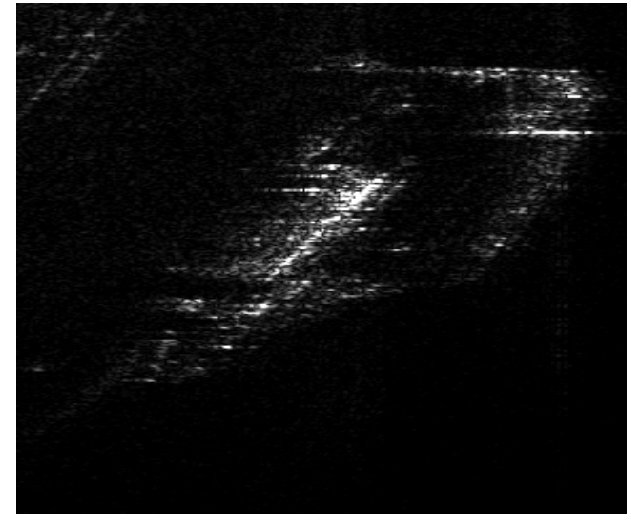

(a) MD-PGA

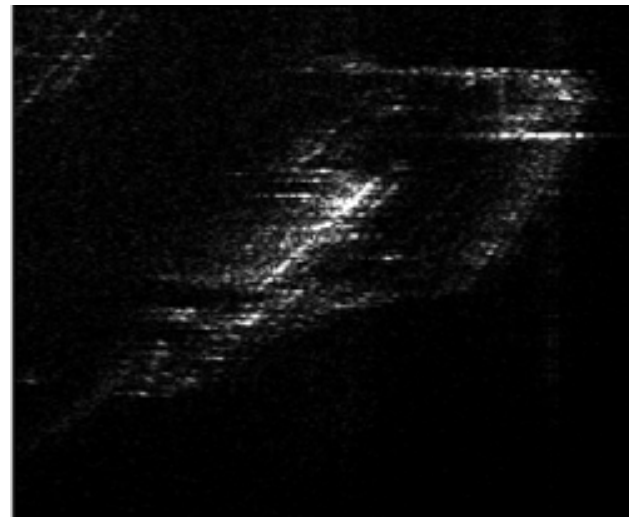

(b) Improved MD-PGA

Fig. (10). Partial enlargement of autofocus imaging results.

Fig. (8)'s entropy decreases, while its contrast ratio increased significantly, so the image quality has been improved obviously.

Range alignment is used as the motion compensation of range translation. The processing results of measured data shows that the imaging quality has been improved a lot, but there are still some obvious defocus phenomena, which need further motion compensation to correct.

Contrast the autofocus Imaging results, Fig. (9a) - entropy: 10.6567,contrast ratio: 53.7549; Fig. (9b) - entropy: 10.5778, contrast ratio: 54.8773. It is thus clear that the autofocus imaging results of improved MD-PGA is of smaller entropy and bigger contrast ratio. Besides, their partial enlargement of autofocus imaging results, shown in Fig. (10), reveal that improved MD-PGA autofocus effect is better.

The effect of the correction of wavefront curvature error of autofocus imaging results is shown in Fig. (11). Since there is no obvious geometric distortion phenomenon, we only adopt Space-variant post filtering for image to correct the phase error generated by the wavefront curvature. Compared with Fig. (9b), it is obvious that the focusing effect in Fig. (11) improves, after correcting the wavefront curvature error. Calculate the Entropy and contrast ratio of Fig. (11), and we can learn that,the image entropy decreases 


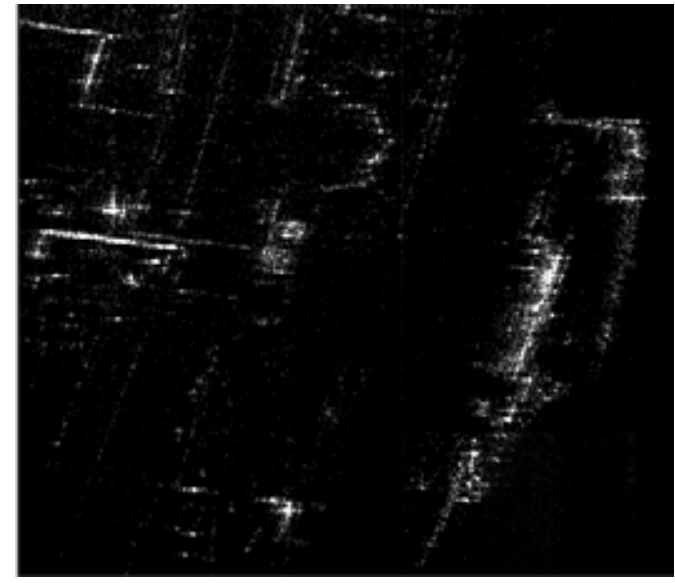

Fig. (11). Wavefront curvature corrected results.

although not significantly, meanwhile the contrast ratio raises markedly from the original 53.7549 to 71.556 .

\section{CONCLUSION}

This paper combining the range alignment technology in ISAR, presents an effective motion compensation method of spotlight SAR, furthermore, it takes improved Space -variant post filtering algorithm to compensate the phase error caused by wavefront curvature. The analysis and processing of the measured data, have verified the feasibility and effectiveness of this method in practical application.

\section{CONFLICT OF INTEREST}

The authors confirm that this article content has no conflict of interest.

\section{ACKNOWLEDGEMENTS}

We would like to express our sincere thanks to The Harbin Institute of Technology for the research opportunity provided.

\section{REFERENCES}

[1] Jakowatz CV, Wahl DE, Eichel PH, et al. Spotlight-Mode synthetic aperture radar: a signal processing approach. Kluwer Boston 1996.

[2] Wan-ming L, Xue-cheng H. High resolution airborne SAR motion compensation based on echo data. J Electr Inform Technol 2004; 26(12): 1908-14.

[3] Wen-wei C, Yi J, Wan-ming L. The implement of real-time motion compensation for high resolution airborne SAR. Signal Process 2006; 22(4): 492-5.

[4] Meng-dao X, Zheng B. Motion parameter esti-mation based SAR imaging. Acta Electron Sin 2001; 29(125): 1824-8.
[5] Wahl DE, Eichel PH, Ghiglia DC. Phase gradient autofocus - a robust tool for high resolution SAR phase correction. IEEE Trans AES 1994; 30(3): 827-35.

[6] Chan HL, Yeo TS. Non-iterative quality phase gradient autofocus (QPGA) algorithm for spotlight SAR imagery. IEEE T Geosci Remote 1998; 36(5): 1531-8.

[7] Abrahamsson R, Li J, Stoica P, Thordarson G. Sensitivity of two autofocus algorithms to spatially variant phase errors. Proc SPIE 2001; 4382: 29-40.

[8] Mancill CE, Swiger JM. A map drift autofocus technique for correcting higher order SAR phase errors. In: $27^{\text {th }}$ Annual Tri-Service Radar Symposium Record. June 1981 Monterey, CA 1981; pp. 391-9.

[9] Bendor GA, Gedra TW. Signal-pass fine-resolution SAR autofocus. In: Proceedings of IEEE National Aerospace and Electronics Conference May1983; NAECON, Dayton, Ohio 1983; pp. 482- 6.

[10] Li FK, Held DN, Curlander JC, Wu C. Doppler parameter estimation for spaceborne synthetic aperture radars. IEEE Trans GRS 1985; 23: 47-56.

[11] Callway TM, Donohoe GW. Sub aperture autofocus for synthetic aperture radar. IEEE Aero El Sys 1994; 30(2): 617-21.

[12] Liu Y, Ng W, Yeo TS, Zhang C. Auto regressive spectral estimation for SAR map-draft auto focusing. In: Asia Pacific Microwave Conference 1997; Hong Kong;1998; pp. 61-4.

[13] Doerry AW. Autofocus correction of SAR images exhibiting excessive residual migration. In: Robert N, Trebits, James L, Kurtz, Eds. Proceedings of SPIE, Bellingham, WA, 2005; 5788: pp. 3445.

[14] Carrara WG, Goodman RS, Majewski RM. Spotlight Synthetic aperture radar signal processing algorithms. Artech House: Boston, 1995.

[15] Zheng B, Meng-dao X, Tong W. Radar imaging technology. Beijing: Publishing House of Electronics Industry 2005.

[16] Martin GD, Dorrry AW. SAR polar format implementation with MATLAB. Technical Rep. SAND 2005-7413, 2005.

[17] Guo-dong W, Yin-qing Z, Chun-sheng L. Polar format processing of high resolution imaging of spaceborne spotlight SAR. Telemetry Remote Control 2003; 24(1): 4-9.

[18] Hai-bin Z, Tao Z, Pei-kun H. A study on frequency domain polar format algorithm of spaceborne spotlight SAR. Modern Radar 2006; 28(1): 28-30.

[19] Haney MW. Real-time acousto-optic processor for spotlight-mode synthetic aperture radar. In: Bendett MP, Butler Jr DH, Prabhakar A, Yang AC, Eds. In: Proceedings of SPIE 1291 Optical and Digital Gallium Arsenide Technologies for Signal Processing Applications. International Society for Optics and Photonics 1990.

[20] Wang Y, Li JW, Chen J, et al. A novel non-interpolation polar format algorithm using non-lineal flight trajectories and autoadaptive PRF technique. Prog Electro Magnet Res 2012; 122(1): 155-73.

[21] Meng-dao X. Study of radar imaging methods based on real data. Xi dian University: China 2002.

[22] Yi-ming Z. Study of new motion compensation methods for SAR/ISAR imaging. Xi' dian University: China 2000.

[23] Ling W. Study on key technologies of inverse synthetic aperture radar imaging. Nanjing University of Aeronautics and Astronautics: China 2006.

[24] Rui J, Dai-yin Z, Zhao-da Z. A novel approach to strip-map SAR autofocus. Acta Aeronautica ET Astronautica Sinica 2010; 31(12): 2386-92.

[25] Xin-hua M, Dai-yin Z, Shao-hua Y, Zhao-da Z. Image domain space-variant post-processing for wavefront curvature correction in polar format spotlight SAR image. Acta Electron Sin 2010; 38(1): 244-50.

(C) Fu-lin et al.; Licensee Bentham Open.

This is an open access article licensed under the terms of the Creative Commons Attribution Non-Commercial License (http://creativecommons.org/licenses/by-nc/3.0/) which permits unrestricted, non-commercial use, distribution and reproduction in any medium, provided the work is properly cited. 